\title{
SENSITIVITY ANALYSIS FOR FORCED RESPONSE IN TURBOMACHINERY USING AN ADJOINT HARMONIC BALANCE METHOD
}

\author{
Anna Engels-Putzka and Christian Frey \\ German Aerospace Center (DLR), Institute of Propulsion Technology \\ Linder Höhe, 51147 Cologne, Germany \\ e-mail: \{Anna.Engels-Putzka,Christian.Frey\}@dlr.de
}

Keywords: Aeroelasticity, Forced Response, Adjoint Methods, Harmonic Balance

\begin{abstract}
The analysis of aeroelastic properties is an important aspect in the design of turbomachinery components. In this study we focus on vibrations caused by the interaction of adjacent blade rows (forced response). This is an inherently unsteady phenomenon. But due to its periodic nature it can be efficiently treated by numerical methods formulated in the frequency domain, e.g. the harmonic balance method.

When going from the analysis of individual designs using CFD to CFD-based optimisation it is desirable to compute also sensitivities of objective functions (targets and restrictions for the optimisation) with respect to design parameters. Since in typical applications the number of design parameters is much larger than the number of objective functions, it is advantageous to use the adjoint method for the computation of these sensitivities.

An adjoint solver based on the harmonic balance method has been implemented in the framework of the flow solver TRACE. This is now extended and used to compute the sensitivities of aeroelastic objective functions to the amplitudes of a harmonic perturbation at the entry or exit of the respective blade row. These sensitivities can be validated by comparing to finite differences obtained from harmonic balance computations with different perturbations. We apply the method to model problems which are representative for turbomachinery configurations.
\end{abstract}




\section{INTRODUCTION}

The design of turbomachinery components is a complex and multi-disciplinary task, and computational methods are more and more extensively used in the design process. Often a compromise between conflicting design goals has to be found. For example, in aircraft propulsion it is on the one hand desired to build compact and light-weight machines, on the other hand also structural properties have to be taken into account and a reduced axial gap between blade rows leads to stronger interactions which could cause unwanted blade vibrations (forced response). Therefore, besides CFD simulations to assess the aerodynamic performance of single blade rows or entire components, also aeroelastic computations are important.

Aeroelastic phenomena in turbomachinery are inherently unsteady, but often of a periodic nature, which makes them attractive for the application of frequency domain methods. These are in most cases computationally more efficient than unsteady methods in time-domain. While some phenomena, e.g. blade flutter, can often be adequately simulated by time-linearized unsteady methods, e.g. the linear harmonic (LH) method [1], there are also important nonlinear effects, in particular for forced response (cf. [2]). An overview on the use of frequency domain methods in turbomachinery applications has been given by He [3] and in [4] Ekici and Huang compare time- and frequency-domain methods.

In our work we focus on the harmonic balance (HB) method as proposed by Hall [5]. It has been applied by Thomas et al. [6,7] to analyse nonlinear effects in aeroelastic configurations. The same approach has been used by Huang and Ekici $[8,9,10]$ and also Gopinath et al. developed a similar time-spectral method [11, 12]. In [13] different variants of HB are reviewed and several applications are presented. The form where the harmonic balance equations are formulated in the frequency domain is termed the nonlinear frequency domain (NLFD) form. It is used e.g. by McMullen et al. [14, 15, 16]. The implementation of a harmonic balance method in the DLR flow solver TRACE, which also uses the NLFD approach, and its application to aeroelastic analysis are described in [17] and [18].

Besides the numerical evaluation of given designs it is - due to increased computational resources - also possible to explore large ranges of designs in CFD-based automatic optimisations. To obtain efficient optimisation algorithms it is useful to also have access to derivatives of the functions which are employed as objectives or constraints in the optimization. There are different possibilities to compute such derivatives. The simplest is to use finite differences, but this requires at least one additional function evaluation for each partial derivative, which can be very expensive. Alternatively, derivatives can be computed directly by a linearisation of the applied computational methods. But since in typical applications the number of design parameters is large compared to the number of objectives, the application of adjoint methods $[19,20]$ is often more efficient than direct linearisation.

While steady adjoint methods are nowadays quite frequently used in the field of turbomachinery design (see e.g. [21, 22]), the application of unsteady adjoint CFD is very limited due to its exceedingly high computational costs, see e.g. [23, 24, 25] and references therein.

A number of adjoint solvers for frequency domain methods have already been developed. Discrete adjoint solvers for LH have been derived "by hand", i.e. by analytic differentiation of the primal code, by Florea and Hall [26] for the Euler equations and by Duta et al. [27, 28] for the RANS equations. Since this approach is very cumbersome for nonlinear methods, many authors apply algorithmic differentiation (AD) techniques in the development of adjoint frequency domain solvers, e.g. Thomas et al. [29] for the HB method of Hall, Huang and Ekici [30] for their implementation of this method, or Mader and Martins [31] for the time-spectral 
method of Gopinath et al. An implementation of an adjoint for the non-linear frequency domain method of McMullen et al. is due to Nadarajah and Jameson [32, 33]. An adjoint method for $\mathrm{HB}$ equations for one harmonic in the time-domain together with an application to a combined aerodynamic and aeroelastic blade optimisation has been presented by He and Wang [34].

The steady adjoint solver in TRACE [35] also employs a discrete adjoint approach, but the linearisation of the residual is obtained by computing finite differences. This approach has now been extended to the frequency domain methods harmonic balance and linear harmonic. The derivation and implementation of the adjoint methods used in this study has been described in detail in [36]. In Section 2 we recall the basic equations and present some recent extensions of the implementation. Afterwards, we apply the methods to two different test cases and compare the results to finite differences from the primal solver (see Section 3).

\section{ADJOINT FREQUENCY DOMAIN METHODS}

\subsection{Harmonic balance method}

The harmonic balance method as implemented in TRACE (see $[17,18]$ ) approximates the time dependent flow solution as a Fourier series with a finite number of harmonics ${ }^{1}$

$$
q(x, t)=\operatorname{Re}\left[\sum_{k=0}^{K} \hat{q}_{k}(x) e^{i k \omega t}\right] .
$$

The Fourier coefficients $\hat{q}_{k}$ are determined by the set of equations

$$
\left.i k \omega \hat{q}_{k}+\widehat{R(q)}\right)_{k}=0, \quad k=0, \ldots, K,
$$

where the Fourier components of the residual are approximated as

$$
\left.\widehat{R(q)}{ }_{k} \approx \mathscr{F}\left(R\left(\mathscr{F}^{-1}(\hat{q})\right)\right)\right|_{k}
$$

with $\mathscr{F}$ denoting the discrete Fourier transform (DFT). This means that the solution $q$ in the time domain is reconstructed at a finite number of sampling points, then the residual is evaluated at these points, and the resulting vector is transformed back into the frequency domain.

The characteristic of the NLFD approach is that the variables which are solved for are the Fourier coefficients $\hat{q}$, while the flow states at the sampling points in time are only computed as intermediate quantities during the solution process. In contrast, in the time-spectral approach, the states at the sampling points are stored and the equations are formulated and solved completely in the time domain.

\subsection{Discrete adjoint approach}

We are now interested in the sensitivity of some functional $I$, which is computed from the solution $q$, to some parameter $\alpha$. We assume that $I$ depends on $\alpha$ only through the Fourier coefficients of $q$, i.e.

$$
\frac{d I}{d \alpha}=\frac{\partial I}{\partial \hat{q}} \frac{d \hat{q}}{d \alpha}
$$

\footnotetext{
${ }^{1}$ In general it could be a combination of such series for several base frequencies, but we restrict the discussion here to a single base frequency, since the adjoint solver has only been developed for this case.
} 
To obtain an equation for $\frac{d \hat{q}}{d \alpha}$ we differentiate (2) with respect to $\alpha$. Defining the harmonic balance residual as $\left.R_{\mathrm{HB}, k}:=i k \omega \hat{q}_{k}+\widehat{R(q)}\right)_{k}$, we get

$$
0=\frac{d R_{\mathrm{HB}, k}}{d \alpha}=\sum_{j}\left(i k \omega \delta_{j k}+\frac{\partial \widehat{R(q)})_{k}}{\partial \hat{q}_{j}}\right) \frac{d \hat{q}_{j}}{d \alpha}+\frac{\partial R_{\mathrm{HB}, k}}{\partial \alpha} .
$$

This is a linear system of equations and we write it briefly as

$$
A \frac{d \hat{q}}{d \alpha}=-\frac{\partial R_{\mathrm{HB}}}{\partial \alpha}
$$

The corresponding adjoint system of equations is then

$$
A^{*} \hat{\psi}=\left(\frac{\partial I}{\partial \hat{q}}\right)^{*}
$$

with the adjoint system matrix given by

$$
A^{*}=\left(\mathscr{F}^{-1}\right)^{*}\left(\operatorname{diag}\left(\left.\frac{\partial R}{\partial q}\right|_{q\left(t_{j}\right)}\right)\right)^{*} \mathscr{F}^{*}-\operatorname{diag}(i k \omega),
$$

where $\operatorname{diag}(\ldots)$ denotes a block diagonal matrix with the corresponding entries on the diagonal and $t_{j}$ are the sampling points for the DFT. The adjoint operator in the frequency domain is defined with respect to the scalar product

$$
\langle\hat{\psi}, \hat{q}\rangle=\operatorname{Re}\langle\hat{\psi}, \hat{q}\rangle_{\mathbb{C}}
$$

This means that we consider the set of (complex) Fourier coefficients as element in a real vector space, which is due to the fact that the transformations $\mathscr{F}$ and $\mathscr{F}^{-1}$ are not linear over the complex numbers.

Using (7), the expression for the sensitivities (4) can be rewritten as

$$
\frac{d I}{d \alpha}=-\hat{\psi}^{*} \frac{\partial R_{\mathrm{HB}}}{\partial \alpha}
$$

which is now independent of $\hat{q}$.

\subsection{Solution method and implementation}

As in the steady adjoint solver in TRACE (see [35]) the discrete adjoint equations (7) are solved by a preconditioned GMRes (Generalised Minimal Residual) algorithm with restarts, as described in [36]. Due to the definition of the scalar product (cf. Eqn. (9)) all "internal" data for the GMRes algorithm is stored as real vectors and matrices. As preconditioner we use either SSOR or ILU(p), i.e. incomplete LU decomposition with variable fill level [37].

The sampling points for the DFT are chosen to be equidistant, i.e. we have $t_{j}=\frac{2 \pi j}{\omega N}$ if $\omega$ is the base frequency and $N$ the number of sampling points. To resolve $K$ higher harmonics, at least $N=2 K+1$ points are needed. But due to the nonlinearity of the residual function, the problem of aliasing can occur [17]. Therefore a higher number of sampling points should be used. Since the residual is a rational function of the flow variables, it is not possible to choose the number of sampling points such that aliasing can be avoided completely. In our computations we take $N=4 K+1$, which is sufficient to resolve cubic terms, so that the remaining errors are of fourth order and should be small in most applications.

The residual Jacobians at the sampling points are computed analogous to the steady case, i.e. finite differences are used for the fluxes while the source terms are differentiated analytically. 


\subsection{Functionals}

The right hand side of (7) is given by the linearisation of the objective functional w.r.t. the state variables. Functionals which are relevant for aeroelastic applications are defined on the blade surfaces. We consider here the modal force for a mode $\Phi$ and frequency $\omega$, which is given by

$$
F_{\text {mod }}=\left\langle\Phi, \hat{p}_{\omega} \vec{n}\right\rangle_{L^{2}}=\int_{\Gamma}\left\langle\Phi, \hat{p}_{\omega} \vec{n}\right\rangle d S
$$

where $\Gamma$ is the blade surface, $\vec{n}$ the surface normal and $\hat{p}_{\omega}$ the Fourier coefficient of the pressure on the blade for the frequency $\omega$. In the context of this study we assume that $\Phi$ and $\vec{n}$ are fixed, so that the linearisation of $F_{\text {mod }}$ depends only on $\hat{p}_{\omega}$ :

$$
d F_{\text {mod }}=\frac{\partial F_{\text {mod }}}{\partial \hat{p}_{\omega}} d \hat{p}_{\omega}
$$

Instead of the modal force itself, which is a complex-valued function, we consider its real and imaginary parts as functionals. In the discrete context, the integral in (11) is evaluated as a sum over the faces on the blade surface:

$$
F_{\text {mod }}=\sum_{\sigma} F_{\text {mod }, \sigma} \quad \text { with } \quad F_{\text {mod }, \sigma}=\hat{p}_{\omega, \sigma}\left\langle\Phi_{\sigma}, \vec{A}_{\sigma}\right\rangle_{\mathbb{C}^{3}},
$$

where $\vec{A}_{\sigma}$ is the face normal vector with $\left|\vec{A}_{\sigma}\right|$ equal to the area of the face $\sigma$. In the following we drop the subscripts $\sigma$ and $\omega$ for simplicity. The real and imaginary parts of $F_{\text {mod }}$ can then be written as

$$
\begin{aligned}
& \operatorname{Re}\left(F_{\text {mod }}\right)=\operatorname{Re}(\hat{p}) A_{\Phi, \operatorname{Re}}+\operatorname{Im}(\hat{p}) A_{\Phi, \operatorname{Im}} \\
& \operatorname{Im}\left(F_{\text {mod }}\right)=\operatorname{Im}(\hat{p}) A_{\Phi, \operatorname{Re}}-\operatorname{Re}(\hat{p}) A_{\Phi, \mathrm{Im}},
\end{aligned}
$$

where $A_{\Phi, \operatorname{Re}}$ and $A_{\Phi, I m}$ are the (real) scalar products of the real and imaginary parts of $\Phi$ with $\vec{A}$, respectively.

For the derivative with respect to the Fourier coefficients of $q$, which is needed for the right hand side of the adjoint equations, we get (compare (12))

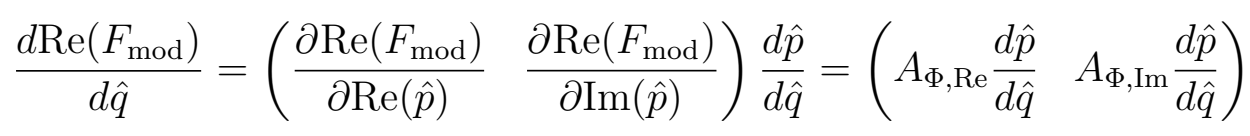

and analogously

$$
\frac{d \operatorname{Im}\left(F_{\mathrm{mod}}\right)}{d \hat{q}}=\left(-A_{\Phi, \operatorname{Im}} \frac{d \hat{p}}{d \hat{q}} \quad A_{\Phi, \operatorname{Re}} \frac{d \hat{p}}{d \hat{q}}\right)
$$

where we have used the fact that $\frac{d \hat{p}}{d \hat{q}}$ is real.

\subsection{Sensitivity evaluation}

To analyse the right hand side of (10) we assume that the flow field $q$ depends on the parameter $\alpha$ only through the prescribed boundary values $q_{\mathrm{bv}}$, i.e. the flow conditions at entry and exit boundaries of the computational domain. Then we get

$$
\frac{d I}{d \alpha}=-\hat{\psi}^{*} \frac{\partial R_{\mathrm{HB}}}{\partial \alpha}=\left(-\hat{\psi}^{*} \frac{\partial R_{\mathrm{HB}}}{\partial q^{\mathrm{ext}}}\right)\left(T_{\mathrm{bv}} \frac{d q_{\mathrm{bv}}}{d \alpha}\right)
$$


where $q^{\text {ext }}$ denotes the ghost cells and $T_{\mathrm{bv}}$ is the inhomogeneous part of the linearised boundary operator, i.e. the linearisation of the boundary update operator

$$
\mathcal{T}:\left(q^{\text {int }}, q_{\text {bv }}\right) \mapsto q^{\text {ext }}
$$

with respect to $q_{\mathrm{bv}}$. The multiplication in (18) is a scalar product, which is defined here - as in (9) - as the real scalar product, i.e. the real part of the standard scalar product for complex vectors.

The implementation of the sensitivity evaluation is similar to the computation of boundary value sensitivities for the steady adjoint solver which is discussed in [38]. The term $\hat{\psi}^{*} \frac{\partial R_{\mathrm{HB}}}{\partial q^{\mathrm{ext}}}$ from (18) is evaluated as $\left(\left(\frac{\partial R_{\mathrm{HB}}}{\partial q^{\mathrm{ext}}}\right)^{*} \hat{\psi}\right)^{*}$ by applying the (adjoint) system matrix to the adjoint solution. The "outer" adjoint is then computed implicitly in the scalar product. The other factor of the product, $T_{\mathrm{bv}} \frac{d q_{\mathrm{bv}}}{d \alpha}$, is computed by applying the (inhomogeneous) linearised boundary conditions to a zero vector. In contrast to the steady case, the boundary conditions here are the gust boundary conditions, where a harmonic disturbance with a specified frequency and (circumferential) wave number is prescribed at the entry or exit of a blade row [39]. Since the parameters $\alpha$ we are interested in are the components of the gust perturbation themselves, $\frac{d q_{\mathrm{bv}}}{d \alpha}$ is trivial. To obtain the result of applying the linearised boundary operator for one parameter, we set the corresponding component of the gust boundary values to unity (with the appropriate de-dimensionalisation) and use these values for the inhomogeneous part of the boundary conditions. Since the gust values are prescribed band-wise, the sensitivities are also computed for each band, which means that the contributions of all faces belonging to a given band are summed up.

\subsection{Linear harmonic method}

The linear harmonic method is obtained if the nonlinear residual is approximated by its linearisation about the time average $\hat{q}_{0}$, which is justified if the amplitudes of the harmonic perturbations $\left(\left|\hat{q}_{k}\right|\right.$ for $\left.k>0\right)$ are small. Then the different harmonics decouple and the flow state is determined by the steady equation $R\left(\hat{q}_{0}\right)=0$ and a linear equation

$$
\left(i k \omega+\left.\frac{\partial R}{\partial q}\right|_{\hat{q}_{0}}\right) \hat{q}_{k}=0
$$

for each $k>0$ [40, 39]. Accordingly, the system matrix for the adjoint LH equations is a block-diagonal matrix with the blocks

$$
A_{k}^{*}=\left(\left.\frac{\partial R}{\partial q}\right|_{\hat{q}_{0}}\right)^{*}-i k \omega .
$$

If gust boundary conditions are applied, (20) takes the form $A_{k} \hat{q}_{k}=S_{k}$, i.e. the boundary conditions lead to an additional source term [39]. This source term depends on the boundary value parameters $\alpha$ and the sensitivities for the adjoint LH method are given by the expression

$$
\frac{d I}{d \alpha}=\hat{\psi}^{*} \frac{\partial S}{\partial \alpha}
$$

where $\hat{\psi}$ is the adjoint solution as before.

The implementation of the adjoint LH method is analogous to adjoint HB, but the setup and application of the system matrix are simplified. Moreover, only the time-average is needed from the primal solution. Another subtle difference is that since the problem is now complex-linear, the complex scalar product can be used and all computations - including the GMRes algorithm - are carried out over the complex numbers. 




Figure 1: CAA Benchmark configuration.

\section{APPLICATION}

In this section we apply the described methods to single blade-row configurations with a prescribed gust perturbation at the entry surface. On the blades an (artificial) mode with the same frequency and phase shift as the gust perturbation is applied. The real and imaginary parts of the modal force for this mode are used as functionals for the adjoint solver and we evaluate the sensitivities with respect to the components $q_{\mathrm{bv}, j}$ of the gust perturbation. These are the real and imaginary parts of the flow parameters at the entry, i.e. the density $\rho$, the velocity components (in cylindrical coordinates) $u_{x}, u_{r}, u_{\theta}$ and the pressure $p$, so that there are in total ten independent parameters.

To obtain approximate derivatives for comparison with the sensitivities computed by the adjoint solver we vary (sequentially) each component of the perturbation at the entry by a constant multiple of the corresponding reference quantity, i.e.

$$
q_{\mathrm{bv}, j} \mapsto q_{\mathrm{bv}, j}+\delta q_{\mathrm{ref}, j},
$$

and perform a harmonic balance (or linear harmonic) simulation with this modified gust perturbation. We choose $\delta=10^{-4}$ as step size, but it has been verified for the used test cases that the result is not sensitive to this choice within a certain range. To compare the two methods for computing sensitivities the relative deviation of a sensitivity $s$ is computed as

$$
\Delta s=\frac{s_{\mathrm{adj}}-s_{\mathrm{FD}}}{s_{\mathrm{FD}}} .
$$

\subsection{Numerical test case}

As a first test case we take a configuration that is based on the "CAA Benchmark Category 3 Problem 1" from [41], which was originally designed as a test for computational aeroacoustics methods. It consists of a cascade of (infinitely thin) flat plates (see Fig. 1). It is treated as a rotational configuration, but with a relatively large radius and therefore a high number of passages (1885). We use a scaled version of the original configuration with radius $R=3 \mathrm{~m}$. The distance between the blades (pitch) is equal to the cord length and the computational domain extends to two times the cord length on both sides. The computational grid for one passage consists of 50000 cells $(500 \times 100 \times 1)$. In circumferential direction periodic boundary conditions are applied.

The steady flow conditions are uniform with density $\rho_{\infty}=1.08 \frac{\mathrm{kg}}{\mathrm{m}^{3}}$ and axial velocity $u_{\infty}=166 \frac{\mathrm{m}}{\mathrm{s}}$, which corresponds to a Mach number of 0.5 . At the entry plane a "vortical 


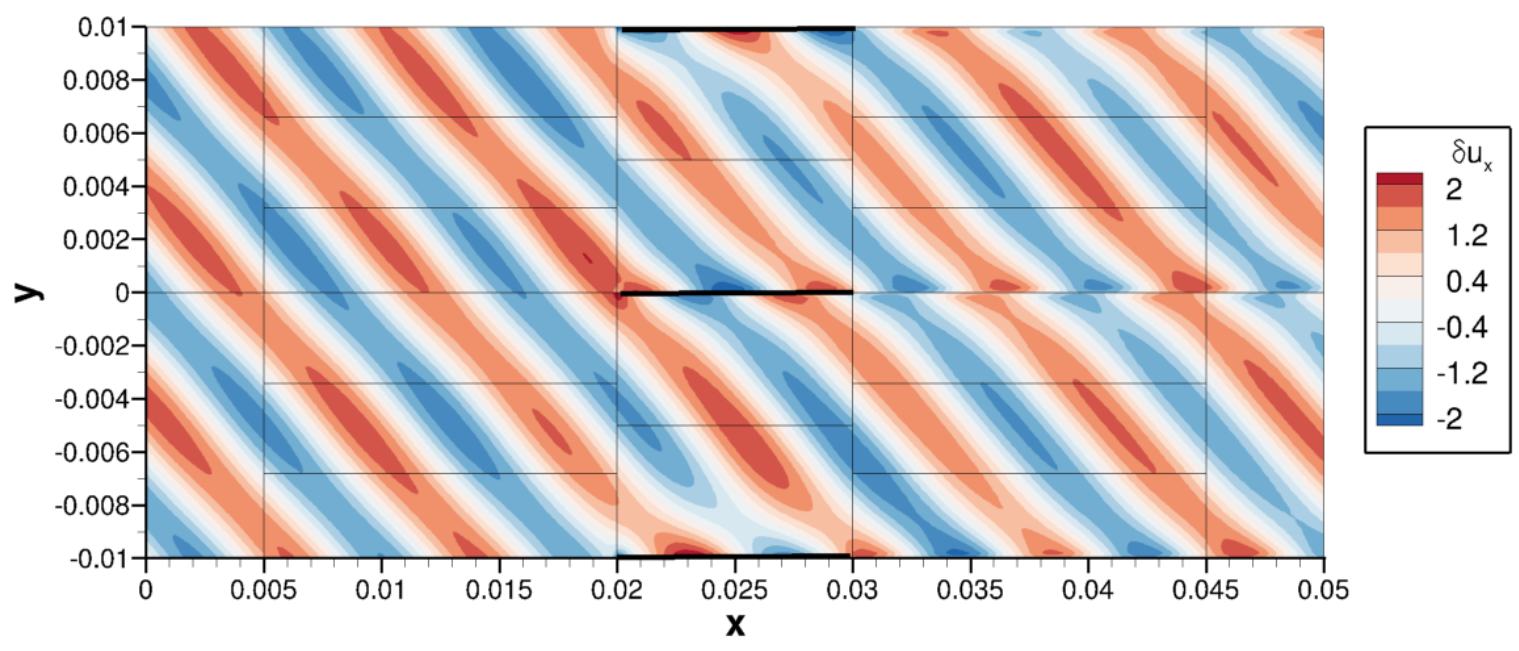

Figure 2: First harmonic of the axial velocity, reconstructed at time $t=0$ from the harmonic balance solution including one higher harmonic.

gust" is prescribed, i.e. a perturbation of the axial and circumferential components of the velocity (real part only). It has a frequency of $20700 \mathrm{~Hz}$ and a circumferential wave number of 2356 , which corresponds to a phase shift (inter-blade phase angle) of 90 degrees, and the magnitude of the perturbation is $0.01 u_{\infty}=1.66 \frac{\mathrm{m}}{\mathrm{s}}$. Figure 2 visualises the flow pattern which is induced by this perturbation. For illustration the blades are drawn with a finite thickness.

The artificial mode applied on the blades in this case corresponds to a torsion. Sensitivities are only computed for the non-trivial components of the gust perturbation, i.e. the axial and circumferential velocity. The reference quantity is $u_{\text {ref }}=338.5 \frac{\mathrm{m}}{\mathrm{s}}$.

Although the configuration is simple, the test case is numerically challenging. The convergence of the pseudo-time solver employed for the harmonic balance and linear harmonic methods is rather slow in this case and a relatively small CFL-number of 3 has to be used. The computations are stopped after 100000 pseudo-time iterations. Figure 3 shows that the values for the modal force are not entirely converged at this point, but the remaining fluctuations are much smaller than the difference between the values for two different gust perturbations.

The adjoint solver does not converge well if the SSOR preconditioner is used. Therefore we tested the newly implemented ILU preconditioner with different levels of fill-in. For lower levels (up to 4) no improvement is observed, while ILU(5) and ILU(6) work well if the restart interval is large enough. In Fig. 4 the convergence histories for different setups are compared. Based on this comparison we chose ILU(5) and a restart interval of 300 for the following computations, since this yields the fastest convergence in terms of iterations as well as with regard to simulation time. Figure 5 shows the first harmonic of the obtained adjoint flow solutions for the modal force (real and imaginary parts).

In tables 1 and 2 the sensitivities of the real and imaginary parts of the modal force with respect to the axial and circumferential velocity components of the gust perturbation computed with the adjoint solver for the harmonic balance and linear harmonic methods are compared to finite differences. The absolute value of the relative deviation is below $5 \%$ in all cases, which indicates that the implementation of the adjoint solver is in principle correct. An exact equality can not be expected, since there are differences between the primal and the adjoint solver e.g. in the implementation of the boundary conditions. For the linear harmonic computations the 


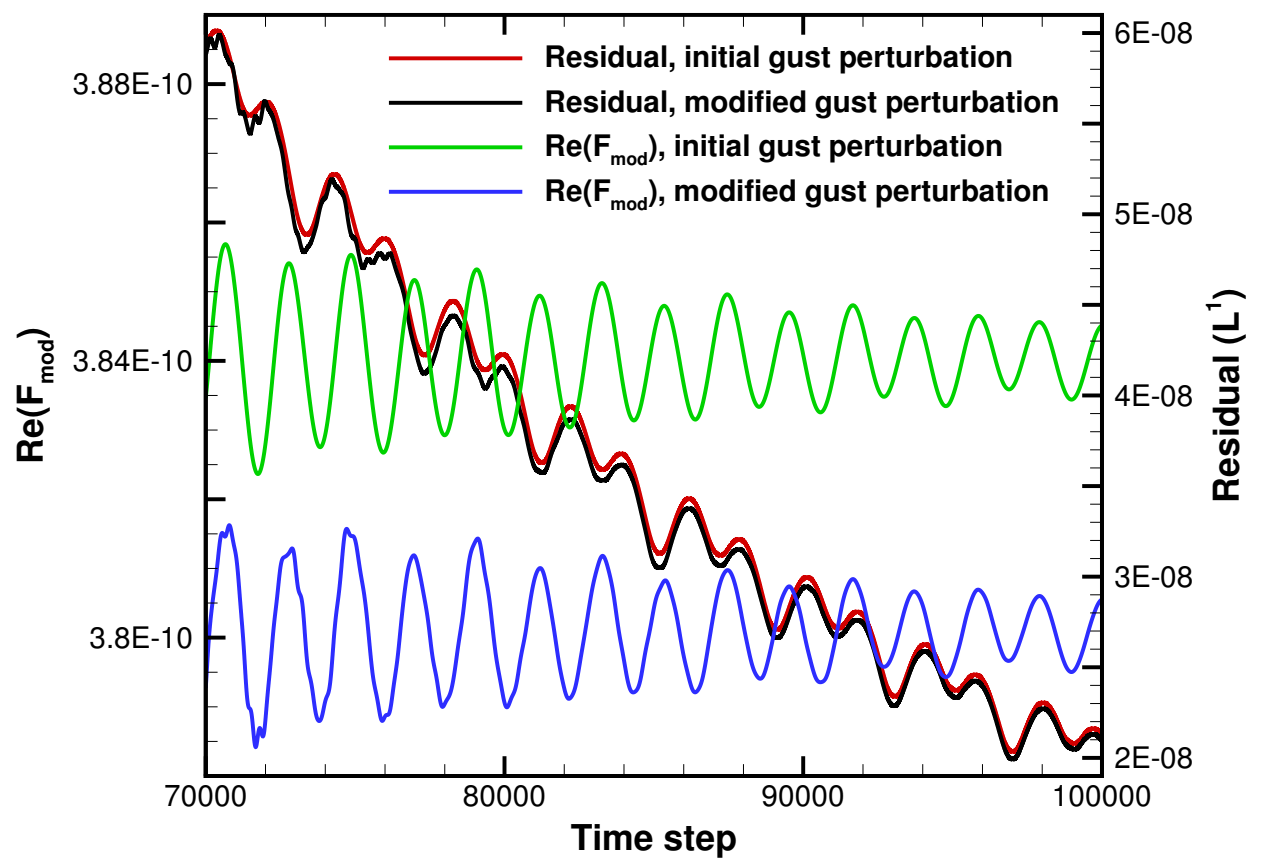

Figure 3: Convergence of the modal force for the initial gust perturbation and the perturbation with modified component $\operatorname{Re}\left(u_{x}\right)$ in relation to the pseudo-time solver residual.
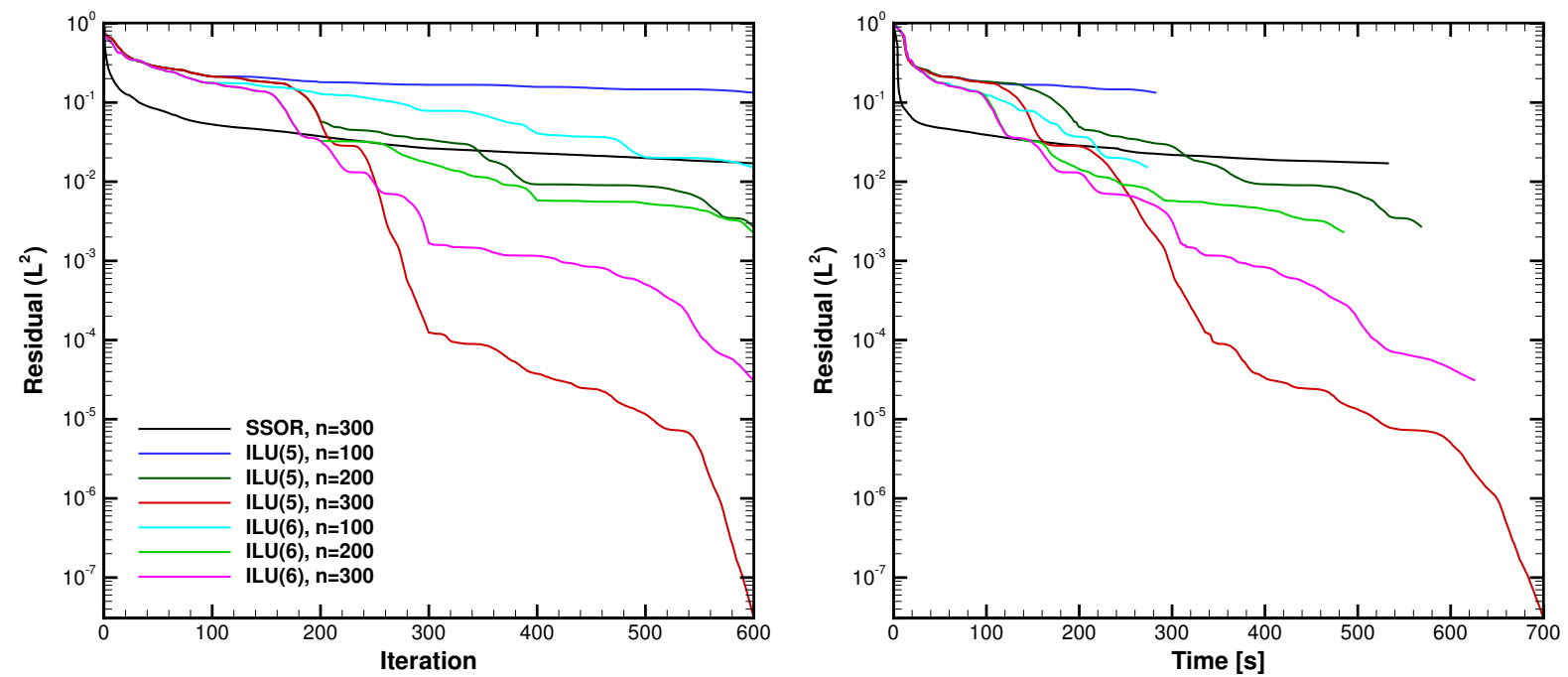

Figure 4: Convergence of the adjoint harmonic balance solver using one higher harmonic for different preconditioners and restart intervals $\mathrm{n}$. 

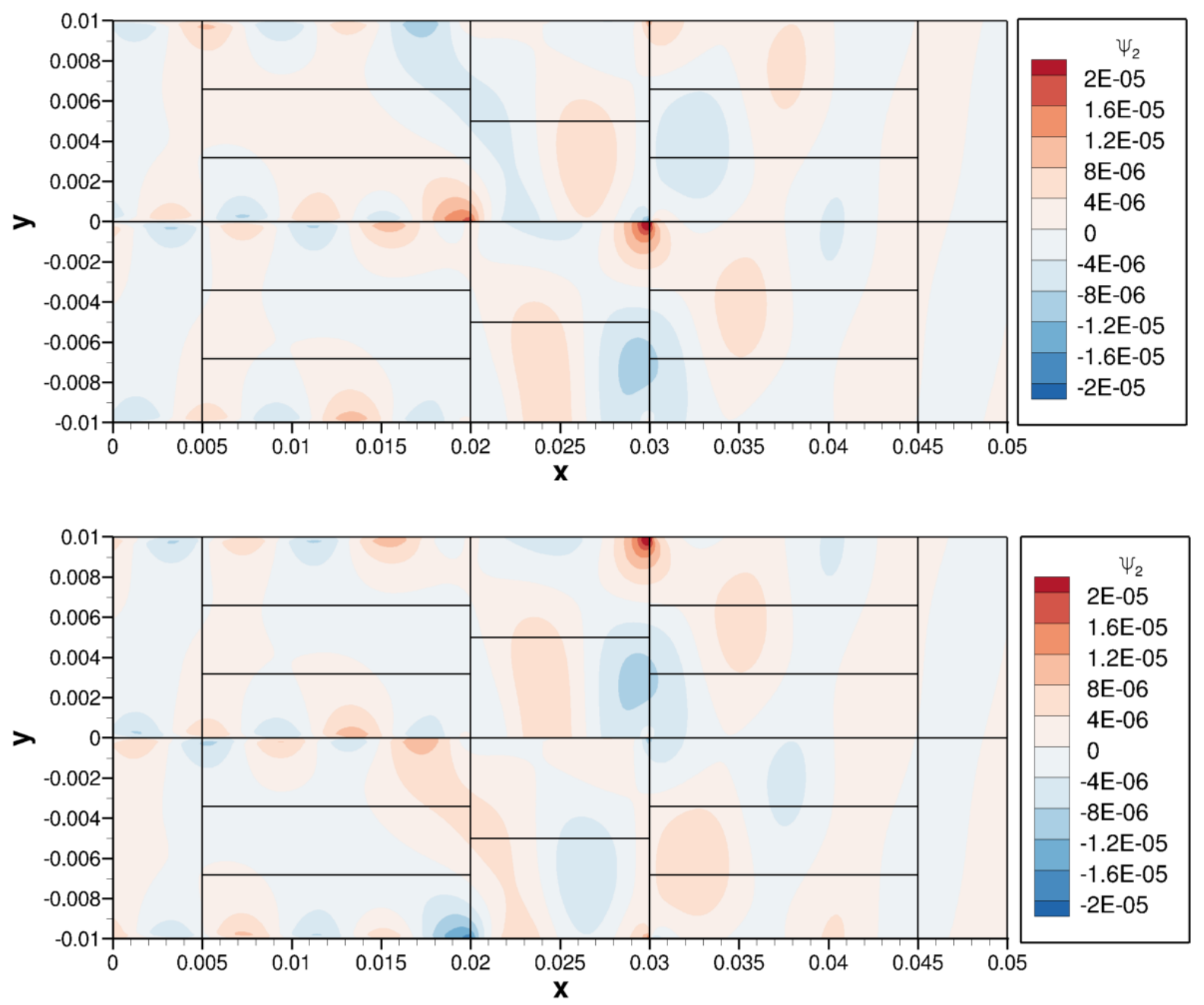

Figure 5: First harmonic of the adjoint solution for the real (top) and imaginary (bottom) parts of the modal force, reconstructed at time $t=0$ from the adjoint harmonic balance solution including one higher harmonic. The component $\psi_{2}$ corresponds to the adjoint momentum in axial direction. 


\begin{tabular}{lccrrrr}
\hline Functional & \multicolumn{3}{c}{$\operatorname{Re}\left(F_{\text {mod }}\right)$} & \multicolumn{3}{c}{$\operatorname{Im}\left(F_{\text {mod }}\right)$} \\
\hline Parameter & \multicolumn{1}{c}{ FD } & \multicolumn{1}{c}{ adjoint } & rel. dev. & \multicolumn{1}{c}{ FD } & \multicolumn{1}{c}{ adjoint } & rel. dev. \\
\hline $\operatorname{Re}\left(u_{x}\right)$ & $-1.171 \cdot 10^{-10}$ & $-1.216 \cdot 10^{-10}$ & $3.88 \%$ & $1.813 \cdot 10^{-10}$ & $1.863 \cdot 10^{-10}$ & $2.73 \%$ \\
$\operatorname{Im}\left(u_{x}\right)$ & $-1.811 \cdot 10^{-10}$ & $-1.859 \cdot 10^{-10}$ & $2.62 \%$ & $-1.166 \cdot 10^{-10}$ & $-1.213 \cdot 10^{-10}$ & $4.07 \%$ \\
$\operatorname{Re}\left(u_{\theta}\right)$ & $-1.165 \cdot 10^{-10}$ & $-1.212 \cdot 10^{-10}$ & $4.00 \%$ & $1.805 \cdot 10^{-10}$ & $1.857 \cdot 10^{-10}$ & $2.91 \%$ \\
$\operatorname{Im}\left(u_{\theta}\right)$ & $-1.803 \cdot 10^{-10}$ & $-1.857 \cdot 10^{-10}$ & $3.02 \%$ & $-1.160 \cdot 10^{-10}$ & $-1.210 \cdot 10^{-10}$ & $4.30 \%$ \\
\hline
\end{tabular}

Table 1: Comparison of sensitivities for the HB method including the zeroth and first harmonic.

\begin{tabular}{lccrrrr}
\hline Functional & \multicolumn{2}{c}{$\operatorname{Re}\left(F_{\text {mod }}\right)$} & \multicolumn{3}{c}{$\operatorname{Im}\left(F_{\text {mod }}\right)$} \\
\hline Parameter & FD & adjoint & rel. dev. & \multicolumn{1}{c}{ FD } & \multicolumn{1}{c}{ adjoint } & \multicolumn{1}{c}{ rel. dev. } \\
\hline $\operatorname{Re}\left(u_{x}\right)$ & $-1.260 \cdot 10^{-10}$ & $-1.211 \cdot 10^{-10}$ & $-3.49 \%$ & $1.837 \cdot 10^{-10}$ & $1.859 \cdot 10^{-10}$ & $1.40 \%$ \\
$\operatorname{Im}\left(u_{x}\right)$ & $-1.837 \cdot 10^{-10}$ & $-1.859 \cdot 10^{-10}$ & $1.19 \%$ & $-1.260 \cdot 10^{-10}$ & $-1.211 \cdot 10^{-10}$ & $-3.74 \%$ \\
$\operatorname{Re}\left(u_{\theta}\right)$ & $-1.255 \cdot 10^{-10}$ & $-1.207 \cdot 10^{-10}$ & $-3.43 \%$ & $1.824 \cdot 10^{-10}$ & $1.854 \cdot 10^{-10}$ & $1.81 \%$ \\
$\operatorname{Im}\left(u_{\theta}\right)$ & $-1.824 \cdot 10^{-10}$ & $-1.854 \cdot 10^{-10}$ & $1.81 \%$ & $-1.255 \cdot 10^{-10}$ & $-1.207 \cdot 10^{-10}$ & $-3.57 \%$ \\
\hline
\end{tabular}

Table 2: Comparison of sensitivities for the LH method including only the first harmonic.

agreement is slightly better than for harmonic balance. The linearity of this method is reflected by the fact that the sensitivities satisfy the relations

$$
\frac{d \operatorname{Re}\left(F_{\text {mod }}\right)}{d \operatorname{Re}(\alpha)}=\frac{d \operatorname{Im}\left(F_{\text {mod }}\right)}{d \operatorname{Im}(\alpha)} \quad \text { and } \quad \frac{d \operatorname{Re}\left(F_{\text {mod }}\right)}{d \operatorname{Im}(\alpha)}=-\frac{d \operatorname{Im}\left(F_{\text {mod }}\right)}{d \operatorname{Re}(\alpha)} .
$$

The differences between the LH and HB results are not very large, which indicates that the nonlinear effects are not very strong in this test case.

\subsection{Q3D slice of a turbine stage}

As a first turbomachinery test case we take a Q3D slice of the rotor from a high pressure turbine stage with subsonic flow conditions (maximum Mach number about 0.78). The wake of the stator is extracted from a steady computation and the circumferential component with wave number $m=-70$, which corresponds to a phase shift of 60 degrees, is prescribed as gust perturbation at the entry of the rotor. The frequency in the relative system of the rotor is $11186.5 \mathrm{~Hz}$. The resulting flow is visualised in Fig. 6. The used artificial mode corresponds to a bending of the blade.

The computational grid is rather coarse with roughly 2000 cells for the rotor row. The forward computations are carried out with a CFL number of 5 and the computations are terminated when the $L^{1}$-residual of the pseudo-time solver has dropped below $10^{-8}$. Figure 7 shows that the modal force is sufficiently well converged at this point. For the adjoint computations the SSOR preconditioner with a relaxation factor of 0.7 is applied and the restart interval is set to 100.

In the adjoint solver, the sensitivities are computed at each restart of the GMRes algorithm, therefore we can also monitor the convergence of these quantities. In Fig. 8 this is shown exemplarily for one component, namely the sensitivity of the real part of the modal force to the real part of the $x$-component of the velocity. It can be seen that after the last restart the sensitivity does not change any more, therefore in the following the adjoint computations are terminated if the (normalized) $L^{2}$-residual of the GMRes solver has dropped below $10^{-8}$. 

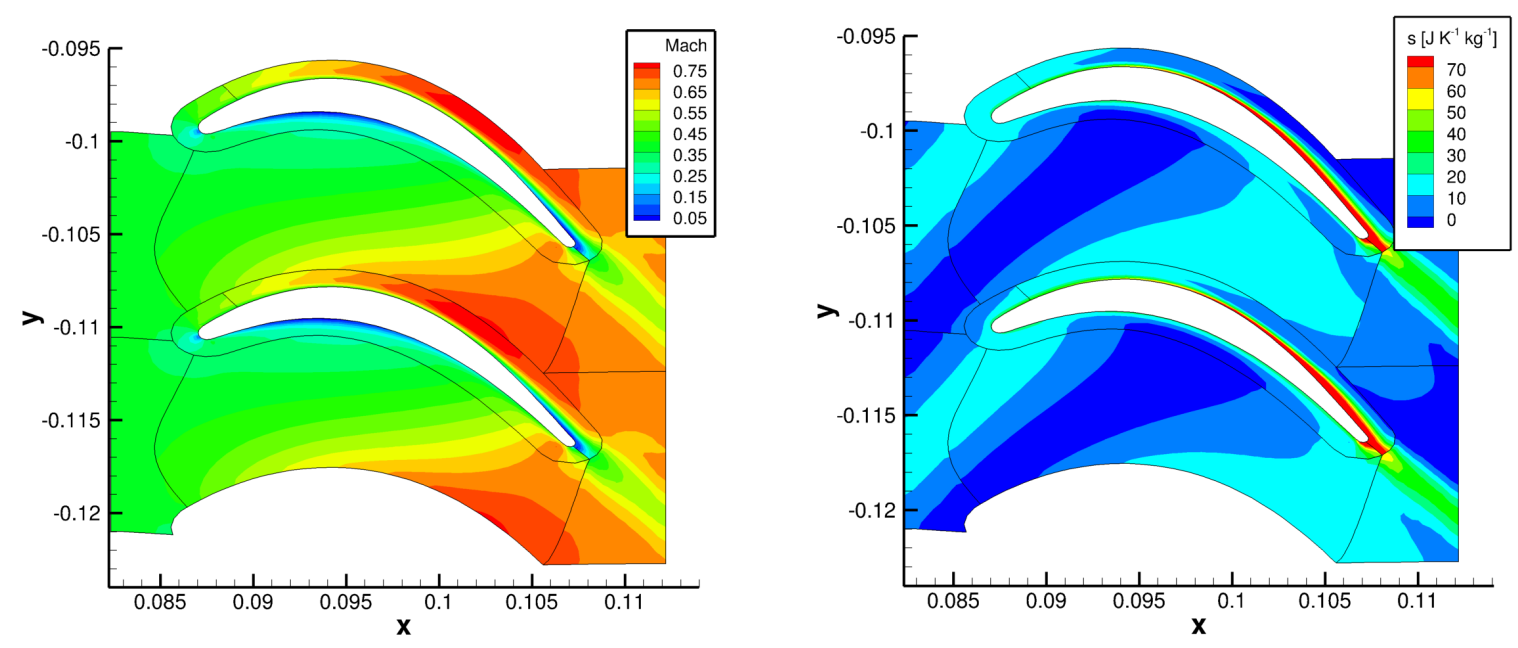

Figure 6: Mach number contours of the time averaged solution (left) and entropy contours of the reconstructed unsteady solution at time $t=0$ (right), both from a harmonic balance computation with four higher harmonics.

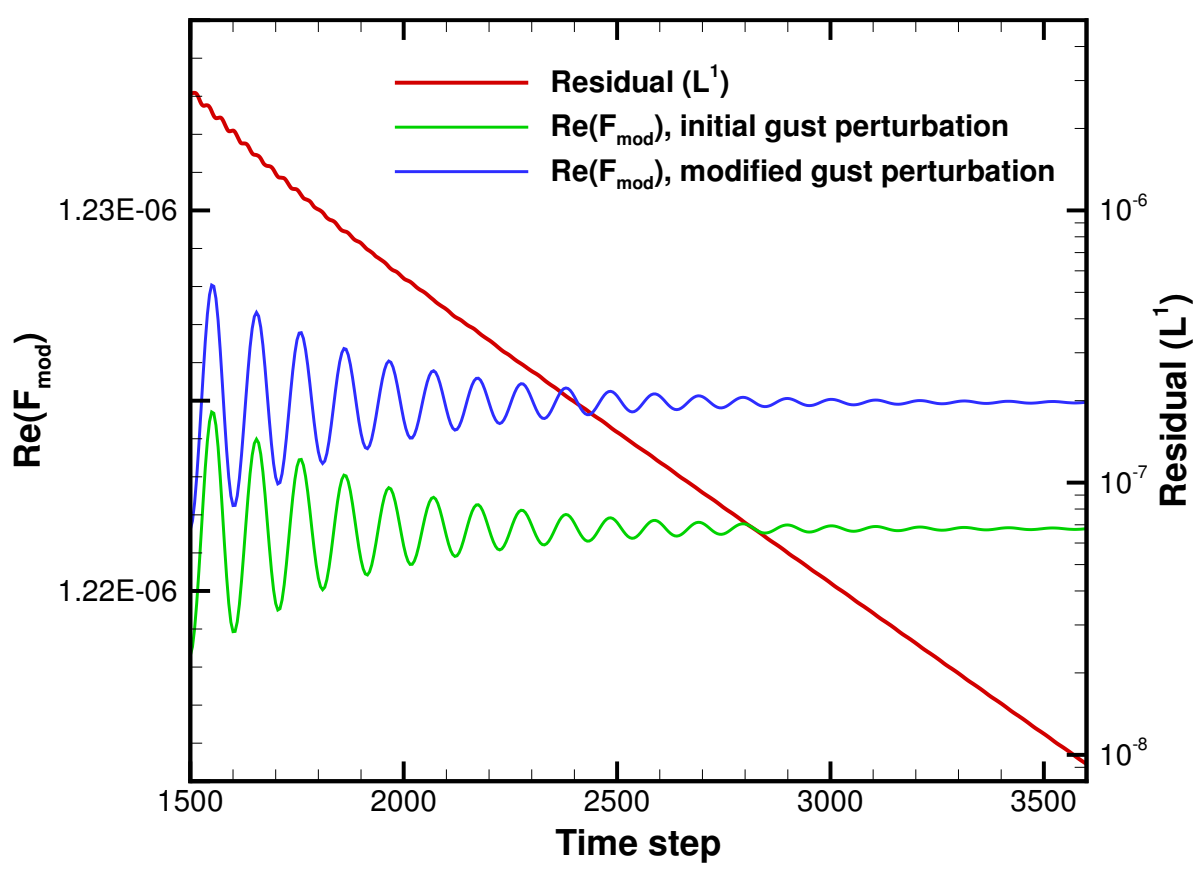

Figure 7: Convergence of the modal force for the initial gust perturbation and the perturbation with modified component $\operatorname{Re}\left(u_{x}\right)$ in relation to the pseudo-time solver residual. 


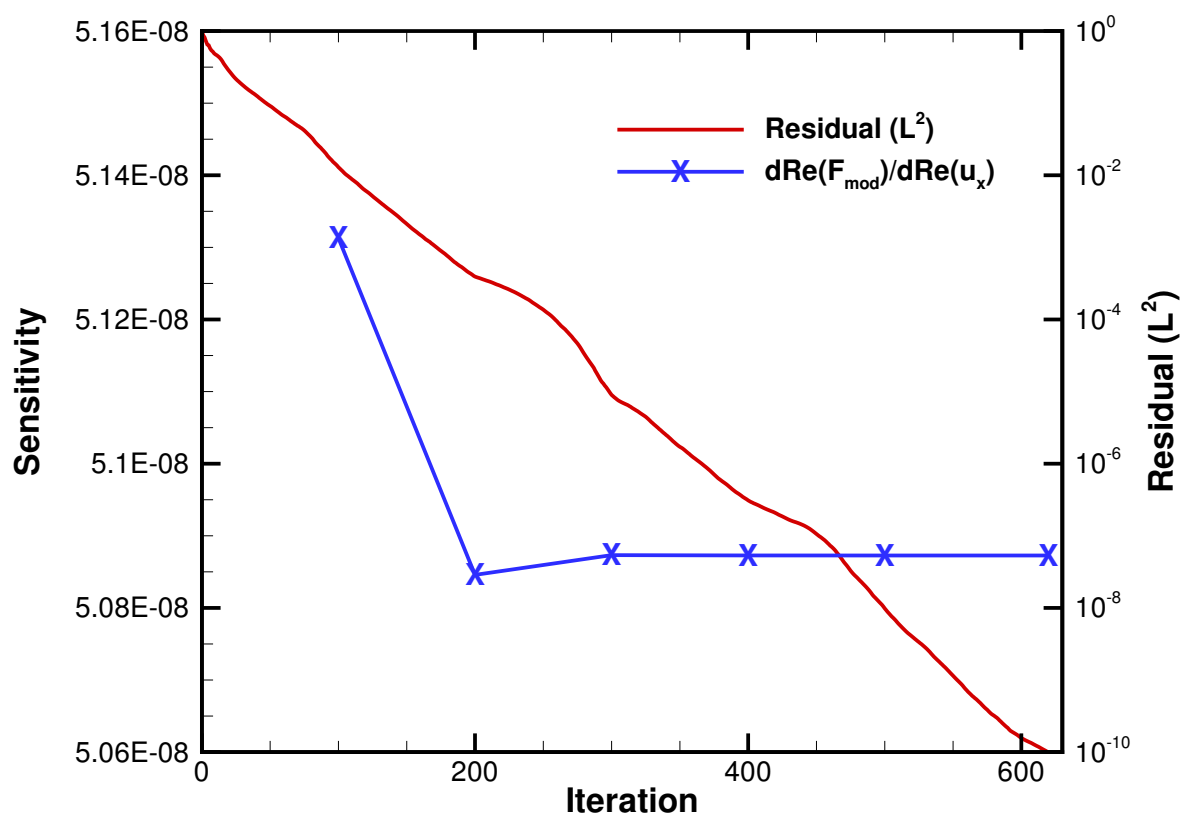

Figure 8: Convergence of sensitivity component in relation to adjoint solver (GMRes) residual.

\begin{tabular}{lrrrrrr}
\hline Functional & \multicolumn{3}{c}{$\operatorname{Re}\left(F_{\text {mod }}\right)$} & \multicolumn{3}{c}{$\operatorname{Im}\left(F_{\text {mod }}\right)$} \\
\hline Parameter & \multicolumn{1}{c}{ FD } & \multicolumn{1}{c}{ adjoint } & rel. dev. & \multicolumn{1}{c}{ FD } & \multicolumn{1}{c}{ adjoint } & rel. dev. \\
\hline $\operatorname{Re}(\rho)$ & $-2.800 \cdot 10^{-5}$ & $-2.880 \cdot 10^{-5}$ & $2.85 \%$ & $1.009 \cdot 10^{-5}$ & $9.980 \cdot 10^{-6}$ & $-1.14 \%$ \\
$\operatorname{Im}(\rho)$ & $-1.139 \cdot 10^{-5}$ & $-1.028 \cdot 10^{-5}$ & $-9.76 \%$ & $-2.769 \cdot 10^{-5}$ & $-2.701 \cdot 10^{-5}$ & $-2.45 \%$ \\
$\operatorname{Re}\left(u_{x}\right)$ & $5.083 \cdot 10^{-8}$ & $5.087 \cdot 10^{-8}$ & $0.08 \%$ & $2.897 \cdot 10^{-8}$ & $2.626 \cdot 10^{-8}$ & $-9.36 \%$ \\
$\operatorname{Im}\left(u_{x}\right)$ & $-2.375 \cdot 10^{-8}$ & $-2.277 \cdot 10^{-8}$ & $-4.15 \%$ & $5.134 \cdot 10^{-8}$ & $5.301 \cdot 10^{-8}$ & $3.25 \%$ \\
$\operatorname{Re}\left(u_{\theta}\right)$ & $-7.081 \cdot 10^{-8}$ & $-7.216 \cdot 10^{-8}$ & $1.90 \%$ & $-3.453 \cdot 10^{-8}$ & $-3.374 \cdot 10^{-8}$ & $-2.28 \%$ \\
$\operatorname{Im}\left(u_{\theta}\right)$ & $2.793 \cdot 10^{-8}$ & $2.756 \cdot 10^{-8}$ & $-1.34 \%$ & $-7.184 \cdot 10^{-8}$ & $-7.620 \cdot 10^{-8}$ & $6.07 \%$ \\
$\operatorname{Re}(p)$ & $7.441 \cdot 10^{-10}$ & $7.514 \cdot 10^{-10}$ & $0.98 \%$ & $3.215 \cdot 10^{-10}$ & $3.124 \cdot 10^{-10}$ & $-2.84 \%$ \\
$\operatorname{Im}(p)$ & $-2.538 \cdot 10^{-10}$ & $-2.543 \cdot 10^{-10}$ & $0.17 \%$ & $7.550 \cdot 10^{-10}$ & $7.759 \cdot 10^{-10}$ & $2.76 \%$ \\
\hline
\end{tabular}

Table 3: Comparison of sensitivities for the HB method including the zeroth and first harmonic.

\begin{tabular}{lrrrrrr}
\hline Functional & \multicolumn{3}{c}{$\operatorname{Re}\left(F_{\text {mod }}\right)$} & \multicolumn{3}{c}{$\operatorname{Im}\left(F_{\text {mod }}\right)$} \\
\hline Parameter & \multicolumn{1}{c}{ FD } & \multicolumn{1}{c}{ adjoint } & rel. dev. & \multicolumn{1}{c}{ FD } & \multicolumn{1}{c}{ adjoint } & rel. dev. \\
\hline $\operatorname{Re}(\rho)$ & $-3.468 \cdot 10^{-5}$ & $-3.465 \cdot 10^{-5}$ & $-0.07 \%$ & $-3.327 \cdot 10^{-6}$ & $-3.330 \cdot 10^{-6}$ & $0.08 \%$ \\
$\operatorname{Im}(\rho)$ & $3.327 \cdot 10^{-6}$ & $3.330 \cdot 10^{-6}$ & $0.08 \%$ & $-3.468 \cdot 10^{-5}$ & $-3.465 \cdot 10^{-5}$ & $-0.07 \%$ \\
$\operatorname{Re}\left(u_{x}\right)$ & $3.546 \cdot 10^{-8}$ & $3.568 \cdot 10^{-8}$ & $0.62 \%$ & $2.594 \cdot 10^{-8}$ & $2.539 \cdot 10^{-8}$ & $-2.12 \%$ \\
$\operatorname{Im}\left(u_{x}\right)$ & $-2.597 \cdot 10^{-8}$ & $-2.539 \cdot 10^{-8}$ & $-2.22 \%$ & $3.540 \cdot 10^{-8}$ & $3.568 \cdot 10^{-8}$ & $0.80 \%$ \\
$\operatorname{Re}\left(u_{\theta}\right)$ & $-5.013 \cdot 10^{-8}$ & $-5.123 \cdot 10^{-8}$ & $2.18 \%$ & $-3.101 \cdot 10^{-8}$ & $-3.137 \cdot 10^{-8}$ & $1.17 \%$ \\
$\operatorname{Im}\left(u_{\theta}\right)$ & $3.101 \cdot 10^{-8}$ & $3.137 \cdot 10^{-8}$ & $1.17 \%$ & $-5.013 \cdot 10^{-8}$ & $-5.123 \cdot 10^{-8}$ & $2.18 \%$ \\
$\operatorname{Re}(p)$ & $5.684 \cdot 10^{-10}$ & $5.719 \cdot 10^{-10}$ & $0.61 \%$ & $3.202 \cdot 10^{-10}$ & $3.229 \cdot 10^{-10}$ & $0.84 \%$ \\
$\operatorname{Im}(p)$ & $-3.202 \cdot 10^{-10}$ & $-3.229 \cdot 10^{-10}$ & $0.84 \%$ & $5.684 \cdot 10^{-10}$ & $5.719 \cdot 10^{-10}$ & $0.61 \%$ \\
\hline
\end{tabular}

Table 4: Comparison of sensitivities for the LH method including only the first harmonic. 


\begin{tabular}{lrrrrrr}
\hline Functional & \multicolumn{3}{c}{$\operatorname{Re}\left(F_{\text {mod }}\right)$} & \multicolumn{3}{c}{$\operatorname{Im}\left(F_{\text {mod }}\right)$} \\
\hline Parameter & \multicolumn{1}{c}{ FD } & \multicolumn{1}{c}{ adjoint } & rel. dev. & \multicolumn{1}{c}{ FD } & \multicolumn{1}{c}{ adjoint } & rel. dev. \\
\hline $\operatorname{Re}(\rho)$ & $-3.476 \cdot 10^{-5}$ & $-3.470 \cdot 10^{-5}$ & $-0.17 \%$ & $-3.345 \cdot 10^{-6}$ & $-3.318 \cdot 10^{-6}$ & $-0.81 \%$ \\
$\operatorname{Im}(\rho)$ & $3.132 \cdot 10^{-6}$ & $3.108 \cdot 10^{-6}$ & $-0.78 \%$ & $-3.522 \cdot 10^{-5}$ & $-3.519 \cdot 10^{-5}$ & $-0.09 \%$ \\
$\operatorname{Re}\left(u_{x}\right)$ & $3.551 \cdot 10^{-8}$ & $3.585 \cdot 10^{-8}$ & $0.97 \%$ & $2.602 \cdot 10^{-8}$ & $2.555 \cdot 10^{-8}$ & $-1.82 \%$ \\
$\operatorname{Im}\left(u_{x}\right)$ & $-2.587 \cdot 10^{-8}$ & $-2.547 \cdot 10^{-8}$ & $-1.57 \%$ & $3.506 \cdot 10^{-8}$ & $3.541 \cdot 10^{-8}$ & $1.00 \%$ \\
$\operatorname{Re}\left(u_{\theta}\right)$ & $-5.018 \cdot 10^{-8}$ & $-5.150 \cdot 10^{-8}$ & $2.64 \%$ & $-3.101 \cdot 10^{-8}$ & $-3.154 \cdot 10^{-8}$ & $1.69 \%$ \\
$\operatorname{Im}\left(u_{\theta}\right)$ & $3.111 \cdot 10^{-8}$ & $3.154 \cdot 10^{-8}$ & $1.39 \%$ & $-4.947 \cdot 10^{-8}$ & $-5.082 \cdot 10^{-8}$ & $2.74 \%$ \\
$\operatorname{Re}(p)$ & $5.687 \cdot 10^{-10}$ & $5.742 \cdot 10^{-10}$ & $0.98 \%$ & $3.205 \cdot 10^{-10}$ & $3.245 \cdot 10^{-10}$ & $1.26 \%$ \\
$\operatorname{Im}(p)$ & $-3.199 \cdot 10^{-10}$ & $-3.239 \cdot 10^{-10}$ & $1.25 \%$ & $5.644 \cdot 10^{-10}$ & $5.697 \cdot 10^{-10}$ & $0.95 \%$ \\
\hline
\end{tabular}

Table 5: Comparison of sensitivities for the HB method including only the first harmonic.

We apply both the harmonic balance and the linear harmonic method, at first using only one harmonic. For the finite differences, all components are varied except for the radial velocity, which is not relevant in a Q3D configuration. The reference quantities are $\rho_{\text {ref }}=0.439 \frac{\mathrm{kg}}{\mathrm{m}^{3}}$, $u_{\text {ref }}=653.3 \frac{\mathrm{m}}{\mathrm{s}}$ and $p_{\text {ref }}=133870 \mathrm{~Pa}$. Tables 3 and 4 show the sensitivities for the two methods computed by finite differences (FD) as described above and by the adjoint solver, and the relative deviation between them. For $\mathrm{LH}$, there is a very good agreement between both solvers. Almost all deviations are below 5\%, most of them even below 1\% (see Tab. 4). For HB the deviations between finite differences and adjoint computations are somewhat larger (see Tab. 3). Most deviations are still below 5\%, but some are also up to $10 \%$ large. These differences could be due to inconsistencies between the two solvers, as mentioned above, but should be further investigated. It can also be observed that the HB sensitivities differ considerably from the LH results. For comparison we also performed a harmonic balance computation with only the first harmonic, i.e. neglecting the coupling between this harmonic and the time-mean solution (zeroth harmonic). Table 5 shows that in this case the results are much closer to those of the LH method, indicating that there is a strong interaction between the zeroth and first harmonic. Comparing the different sensitivity components as in Eqn. (25) we see that they are closer to a linear behaviour than in the coupled computation, but the nonlinearity is still present, although there is only one solution component. A comparison of all methods is shown in Fig. 9 for the sensitivities w.r.t. the angular component of the velocity.

We also investigated the effect of including higher harmonics in the computations. Since only the first harmonic is used for the evaluation of the objective functionals, there is only an indirect influence of higher harmonics through their interaction with the first, and it turns out that the impact on the modal force - and therefore the sensitivities - is indeed small. In Fig. 10 the values of the real and imaginary parts of the modal force are shown over the number of higher harmonics included in the HB computation and it can be seen that only the second harmonic yields a significant contribution. The convergence of the GMRes solver becomes slower if more harmonics are included, as can be seen in Fig. 11, but we were able to obtain converged solutions for all sets of harmonics considered here. The agreement between forward and adjoint results is qualitatively similar to the case with only one higher harmonic, although the maximal deviations become somewhat larger. 


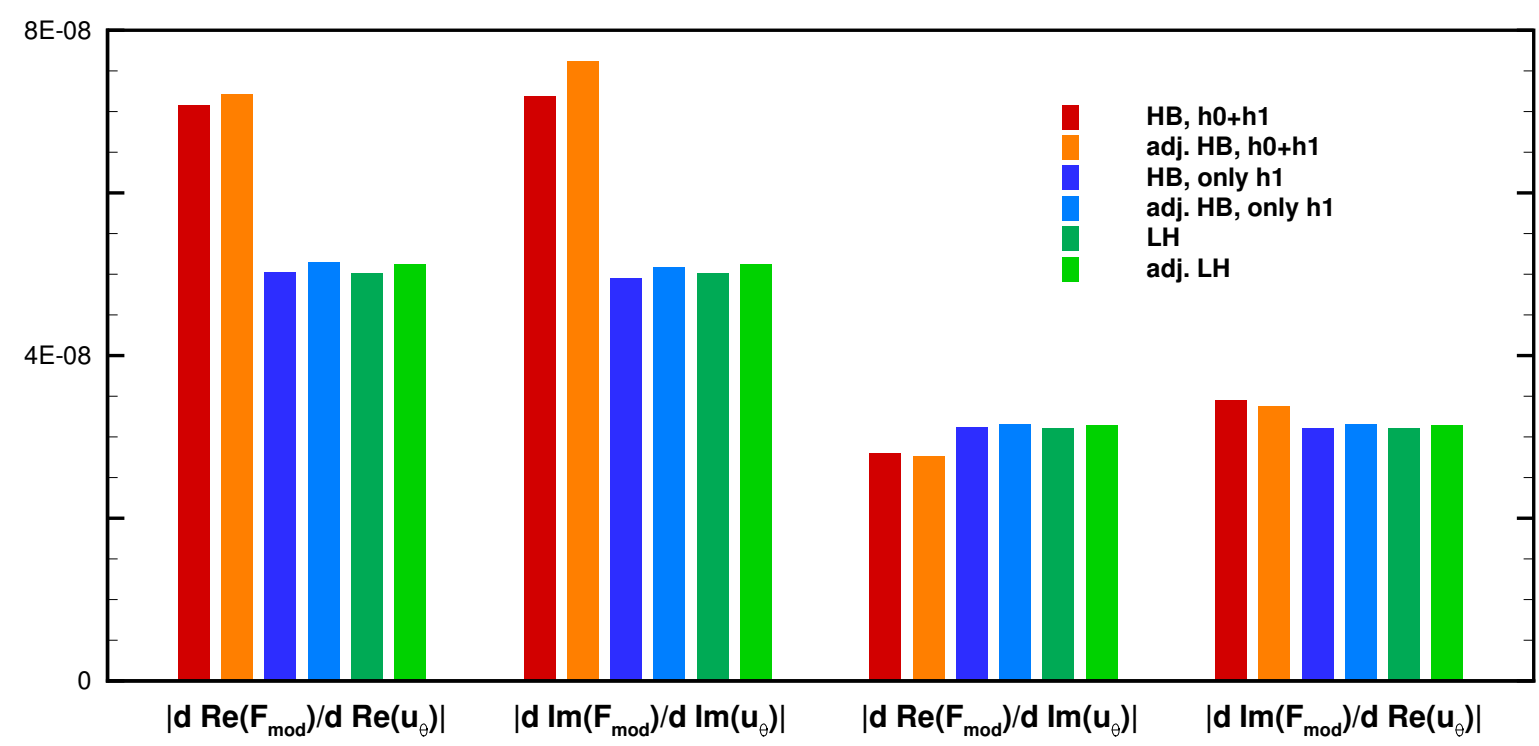

Figure 9: Comparison of four sensitivity components for different methods

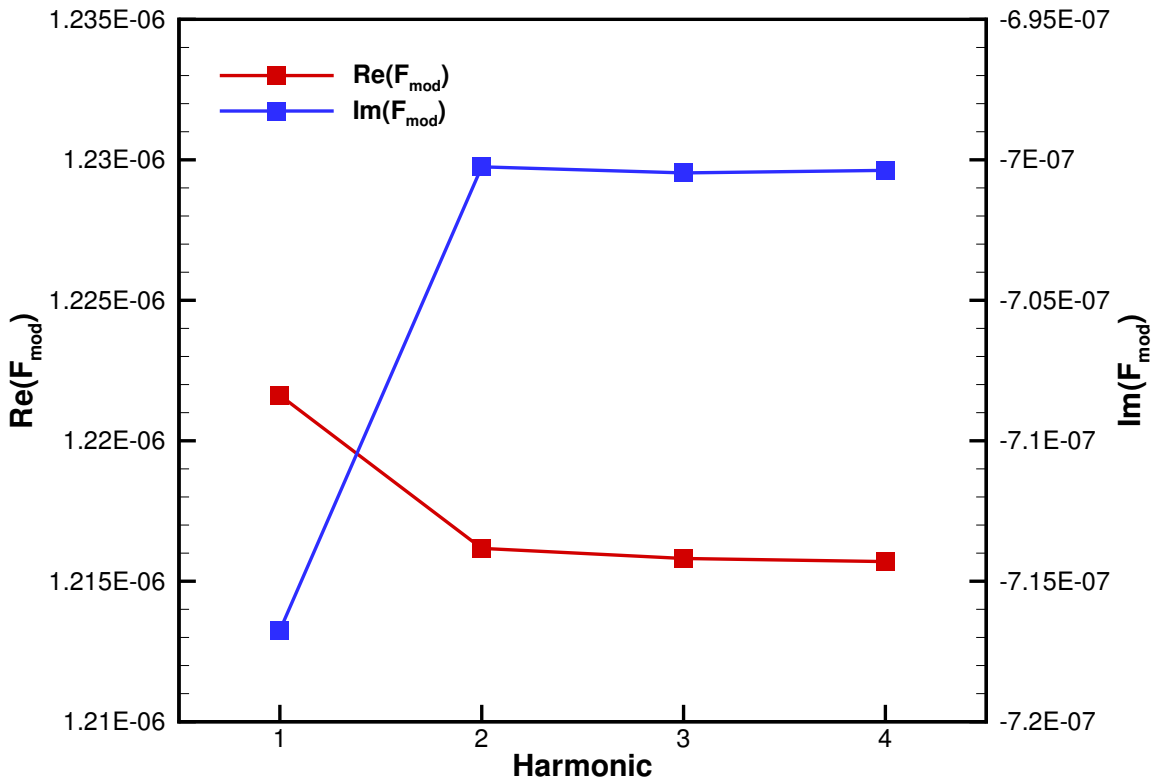

Figure 10: Real and imaginary parts of modal force for harmonic balance computations with different sets of harmonics ("Harmonic" is the order of the highest harmonic which is included). 


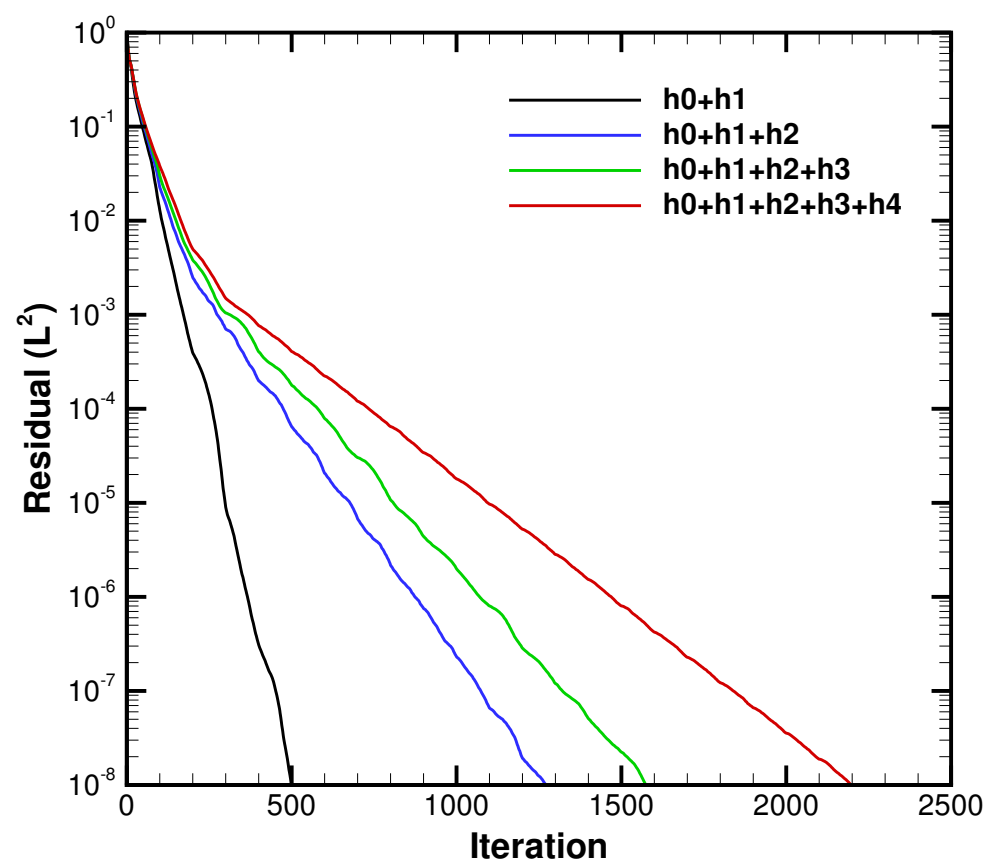

Figure 11: Convergence of the adjoint solver for the functional $\operatorname{Re}\left(F_{\bmod }\right)$ with different sets of harmonics included.

\section{CONCLUSION}

We have described the development and implementation of an adjoint solver for frequency domain methods (harmonic balance and linear harmonics) within an industrial turbomachinery CFD solver. It is based on the extension of an existing steady adjoint solver. In particular, the implementation of the modal force on blades as a functional for aeroelastic applications and the evaluation of sensitivities with respect to the components of a flow perturbation at inflow or outflow boundaries have been discussed. The method has been extensively tested on Q3D configurations which are representative for turbomachinery applications. The overall good agreement of the sensitivities obtained by the adjoint solver with finite differences shows the validity of the approach. The reasons for some larger deviations have to be investigated in the future. Also the extension to 3D configurations has to be tested in detail.

Another important issue for future work is the coupling of different blade rows, since we are interested in the influence of the geometry of a neighbouring blade row on e.g. the modal forces for the blade row under consideration. In the current process, where the influence of e.g. a stator wake on the subsequent blade row is simulated only for selected wave numbers, the next step would be to use the sensitivities from the adjoint harmonic balance solver as input, i.e. objective functional, for a following steady adjoint computation. This would then give the geometric sensitivities for the upstream blade row. To obtain an adjoint process for a complete forcedresponse analysis using harmonic balance, the blade-row coupling and a geometric sensitivity evaluation - analogous to the steady adjoint solver - have to be implemented within the adjoint HB solver.

Acknowledgements The authors would like to thank J. Backhaus and H.-P. Kersken for helpful discussions. 


\section{REFERENCES}

[1] K. C. Hall and E. F. Crawley, "Calculation of unsteady flows in turbomachinery using the linearized Euler equations," AIAA Journal, vol. 27, no. 6, pp. 777-787, 1989.

[2] R. Kielb, "CFD for turbomachinery unsteady flows - an aeroelastic design perspective," in Aerospace Sciences Meetings, American Institute of Aeronautics and Astronautics, Jan. 2001.

[3] L. He, "Fourier methods for turbomachinery applications," Progress In Aerospace Sciences, vol. 46, pp. 329-341, Nov. 2010.

[4] K. Ekici and H. Huang, "An assessment of frequency-domain and time-domain techniques for turbomachinery aeromechanics," in 30th AIAA Applied Aerodynamics Conference 2012, pp. 1807 1823, June 2012.

[5] K. C. Hall, J. P. Thomas, and W. S. Clark, "Computation of unsteady nonlinear flows in cascades using a harmonic balance technique," AIAA Journal, vol. 40, pp. 879-886, May 2002.

[6] J. P. Thomas, E. H. Dowell, and K. C. Hall, "Nonlinear inviscid aerodynamic effects on transonic divergence, flutter, and limit-cycle oscillations," AIAA Journal, vol. 40, pp. 638-646, Apr. 2002.

[7] J. P. Thomas, E. H. Dowell, and K. C. Hall, "Modeling viscous transonic limit-cycle oscillation behavior using a harmonic balance approach," Journal of Aircraft, vol. 41, no. 6, pp. 1266-1274, 2004.

[8] H. Huang and K. Ekici, "A harmonic balance method for the analysis of unsteady flows in cascades," in 47th AIAA/ASME/SAE/ASEE Joint Propulsion Conference and Exhibit, American Institute of Aeronautics and Astronautics, July 2011.

[9] H. Huang and K. Ekici, "An efficient harmonic balance method for unsteady flows in cascades," Aerospace Science and Technology, vol. 29, no. 1, pp. 144-154, 2013.

[10] H. Huang and K. Ekici, "Stabilization of high-dimensional harmonic balance solvers using time spectral viscosity," AIAA Journal, vol. 52, no. 8, pp. 1784-1794, 2014.

[11] A. Gopinath and A. Jameson, "Time spectral method for periodic unsteady computations over twoand three- dimensional bodies," in Proceedings of the 43rd AIAA Aerospace Sciences Meeting \& Exhibit, 2005.

[12] E. van der Weide, A. Gopinath, and A. Jameson, "Turbomachinery applications with the time spectral method," in 35th AIAA Fluid Dynamics Conference and Exhibit, AIAA paper 05-4905, American Institute of Aeronautics and Astronautics, June 2005.

[13] K. C. Hall, K. Ekici, J. P. Thomas, and E. H. Dowell, "Harmonic balance methods applied to computational fluid dynamics problems," International Journal of Computational Fluid Dynamics, vol. 27, no. 2, pp. 52-67, 2013.

[14] M. McMullen, A. Jameson, and J. Alonso, "Acceleration of convergence to a periodic steady state in turbomachinery flows," in AIAA 39th Aerospace Sciences Meeting, AIAA paper 01-0152, American Institute of Aeronautics and Astronautics, Jan. 2001.

[15] M. McMullen, A. Jameson, and J. Alonso, "Application of a non-linear frequency domain solver to the Euler and Navier-Stokes equations," in AIAA 40th Aerospace Sciences Meeting, AIAA paper 02-0120, American Institute of Aeronautics and Astronautics, Jan. 2002. 
[16] M. S. McMullen, The Application of Non-Linear Frequency Domain Methods to the Euler and Navier-Stokes Equations. PhD thesis, Stanford University, 2003.

[17] C. Frey, G. Ashcroft, H.-P. Kersken, and C. Voigt, "A harmonic balance technique for multistage turbomachinery applications," in Proceedings of ASME Turbo Expo 2014, 2014.

[18] G. Ashcroft, C. Frey, and H.-P. Kersken, "On the development of a harmonic balance method for aeroelastic analysis," in 6th European Conference on Computational Fluid Dynamics (ECFD VI), 2014.

[19] A. Jameson, "Aerodynamic design via control theory," Journal of Scientific Computing, vol. 3, no. 3, pp. 233-260, 1988.

[20] M. B. Giles and N. A. Pierce, "An introduction to the adjoint approach to design," Flow, Turbulence and Combustion, vol. 65, pp. 393-415, 2000.

[21] D. X. Wang and L. He, "Adjoint aerodynamic design optimization for blades in multistage turbomachines-part I: Methodology and verification," Journal of Turbomachinery, vol. 132, no. 2, p. 021011, 2010.

[22] D. X. Wang, L. He, Y. S. Li, and R. G. Wells, "Adjoint aerodynamic design optimization for blades in multistage turbomachines-part II: Validation and application," Journal of Turbomachinery, vol. 132, no. 2, p. 021012, 2010.

[23] S. K. Nadarajah and A. Jameson, "Optimum shape design for unsteady flows with time-accurate continuous and discrete adjoint methods," AIAA Journal, vol. 45, pp. 1478-1491, July 2007.

[24] E. J. Nielsen and B. Diskin, "Discrete adjoint-based design for unsteady turbulent flows on dynamic overset unstructured grids," AIAA Journal, vol. 51, pp. 1355-1373, June 2013.

[25] G. Ntanakas and M. Meyer, “Towards unsteady adjoint analysis for turbomachinery applications," in Proceedings of the 6th. European Conference on Computational Fluid Dynamics - ECFD VI, pp. 5071-5081, 2014.

[26] R. Florea and K. Hall, "Sensitivity analysis of unsteady inviscid flow through turbomachinery cascades," AIAA Journal, vol. 39, pp. 1047-1056, June 2001.

[27] M. C. Duta, M. B. Giles, and M. S. Campobasso, "The harmonic adjoint approach to unsteady turbomachinery design," International Journal for Numerical Methods in Fluids, vol. 40, no. 3-4, pp. 323-332, 2002.

[28] M. Duta, M. Campobasso, M. Giles, and L. Lapworth, "Adjoint harmonic sensitivities for forced response minimization," Journal of Engineering for Gas Turbines and Power, vol. 128, pp. 183189, Jan. 2006.

[29] J. Thomas, K. Hall, and E. Dowell, "Discrete adjoint approach for modeling unsteady aerodynamic design sensitivities," AIAA Journal, vol. 43, pp. 1931-1936, Sep. 2005.

[30] H. Huang and K. Ekici, "A discrete adjoint harmonic balance method for turbomachinery shape optimization," Aerospace Science and Technology, vol. 39, pp. 481-490, 2014.

[31] C. Mader and J. Martins, "Derivatives for time-spectral computational fluid dynamics using an automatic differentiation adjoint," AIAA Journal, vol. 50, no. 12, pp. 2809-2819, 2012.

[32] S. K. Nadarajah, M. S. McMullen, and A. Jameson, "Aerodynamic shape optimization for unsteady three-dimensional flows," International Journal of Computational Fluid Dynamics, vol. 20, pp. 533-548, Sep. 2006. 
[33] S. K. Nadarajah and A. Jameson, "Optimum shape design for unsteady three-dimensional viscous flows using a nonlinear frequency-domain method," Journal of Aircraft, vol. 44, no. 5, pp. 15131527, 2007.

[34] L. He and D. X. Wang, "Concurrent blade aerodynamic-aero-elastic design optimization using adjoint method," Journal of Turbomachinery, vol. 133, p. 011021, Jan. 2011.

[35] C. Frey, D. Nürnberger, and H.-P. Kersken, "The discrete adjoint of a turbomachinery RANS solver," in Proceedings of ASME-GT2009, 2009.

[36] A. Engels-Putzka and C. Frey, "Adjoint harmonic balance method for forced response analysis in turbomachinery," in Proceedings of the VI International Conference on Coupled Problems in Science and Engineering, pp. 645-656, 2015.

[37] Y. Saad, Iterative methods for sparse linear systems. 2nd ed. SIAM Society for Industrial and Applied Mathematics, Philadelphia., 2003.

[38] A. Engels-Putzka and C. Frey, "Adjoint mesh deformation and adjoint-based sensitvitities with respect to boundary values," in ECCOMAS 2012 - European Congress on Computational Methods in Applied Sciences and Engineering, e-Book Full Papers, pp. 1789-1808, 2012.

[39] C. Frey, G. Ashcroft, H.-P. Kersken, and C. Weckmüller, "Advanced numerical methods for the prediction of tonal noise in turbomachinery - Part II: Time-linearized methods," Journal of Turbomachinery, vol. 136, no. 2, p. 021002, 2013.

[40] H.-P. Kersken, C. Frey, C. Voigt, and G. Ashcroft, "Time-linearized and time-accurate 3D RANS methods for aeroelastic analysis in turbomachinery," Journal of Turbomachinery, vol. 134, no. 5, p. 051024, 2012.

[41] C. K. W. Tam and J. C. Hardin, eds., Second Computational Aeroacoustics (CAA) Workshop on Benchmark Problems, NASA Conference Publication 3352, NASA Langley Research Center, June 1997. 